EDITORIAL

\title{
Reflexões críticas sobre os três erres, ou os periódicos brasileiros excluídos
}

\author{
Mauricio Rocha e Silva
}

Recentemente CLINICS propôs para debate a idéia de que o QUALIS 2010 era passível de aperfeiçoamento, através do conceito dos três erres (Remover periódicos de revisão, Reconhecer outras métricas de avaliação, Reavaliar periódicos brasileiros). ${ }^{1}$ Submetido ao debate por pares por ocasião do II Seminário Satélite para Editores Plenos, (ABEC Novembro de 2010), concluiu-se que idealmente haveria que focalizar num único erre, a saber, o reconhecimento por parte da CAPES para 2013 das métricas de avaliação SCImago (cites/document) e SCIELO (Impact Factor).

Já havíamos demonstrado anteriormente que o Impact Factor ISI THOMSON, única métrica reconhecida pela CAPES é praticamente idêntico ao cites/document da SCIMAGO. A correlação geral por amostragem entre os dois índices é maior que 0,9 e o coeficiente angular é indistinguível da unidade. A recente divulgação da coleção 2009 do SCImago Journal \& Country Rank ${ }^{2}$ junta-se aos já divulgados Fatores de Impacto JCR-ISI e SCIELO para aquele ano e permite o cotejo em tempo real dos três índices. Salientamos que este cotejo não se aplica a qualquer tabela QUALIS, pois a Tabela 2010 já é história e a Tabela 2013 será decidida no futuro. Em outras palavras, o cotejo que se segue, relativo ao ano 2009, é oferecido como base argumentativa adequada para reivindicar a correção de curso relativa à exclusão dos índices SCImago e SCIELO.

Vamos pois ao cenário 2009 como simulação de uma hipotética tabela Qualis: na versão 2009 do Journal of Citations Report - ISI a representação brasileira saltou de 31 a 71 periódicos; já no SCImago Journal Ranking essa representação manteve-se constante em 235 periódicos. Existem pois 164 periódicos brasileiros (138 com impacto maior que zero) ausentes da tabela JCR-ISI. Mantida a norma Qualis aplicada em 2010, estes 138 periódicos cairiam nas categorias "sem fator de impacto". Mas a Figura 1 mostra a identidade entre ISI e SCImago para as 64 revistas incluídas nos dois índices: o coeficiente angular unitário e o elevado coeficiente de correlação significam que, conhecendo-se um dos índices, pode-se estimar o outro com $95 \%$ de probabilidade de errar por menos de $5 \%$.

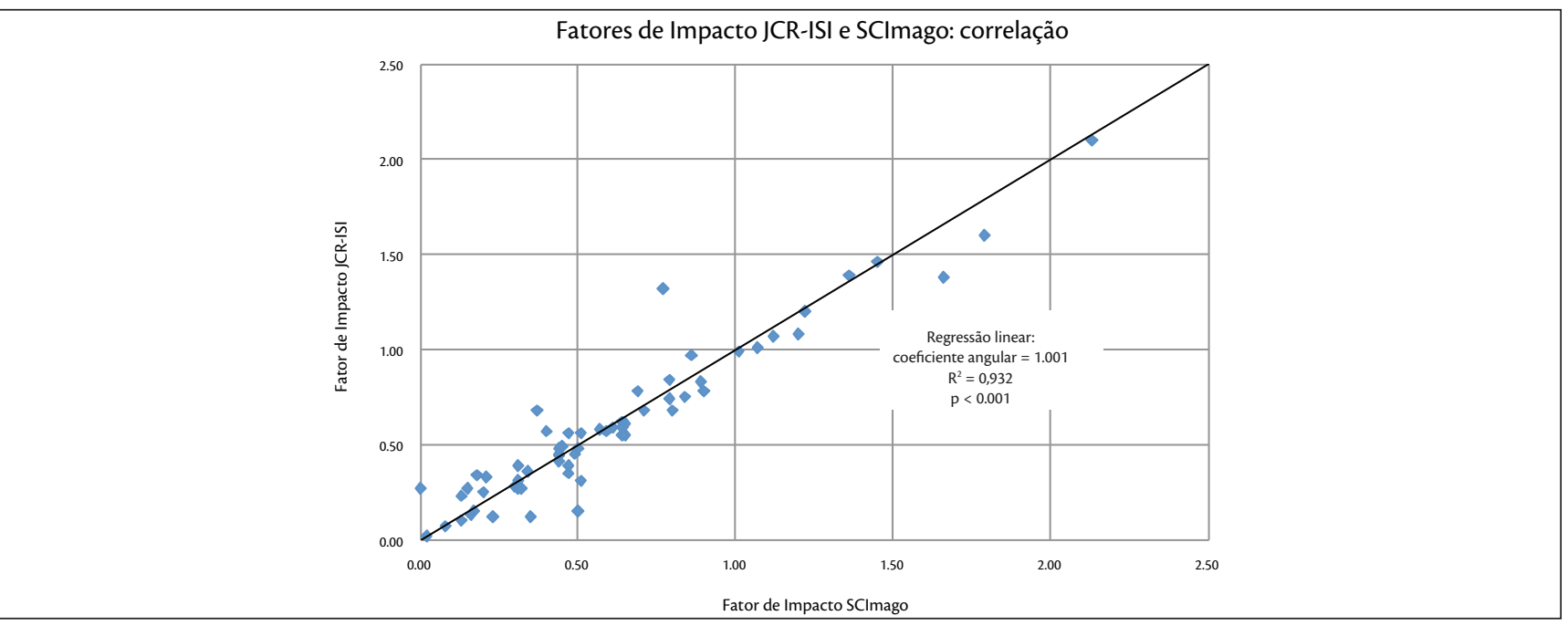

Figura 1. Correlação entre fatores do impacto J. Citation Reports (ISI - Thomson) e SCImago (2009) para 64 periódicos brasileiros representados nos dois índices com valores maiores que zero. A identidade entre métricas é praticamente absoluta. O coeficiente angular indica um ângulo de $45^{\circ}$ (significando identidade) e a correlação $\left(R^{2}=0,932 ; p<0.001\right)$ é quase perfeita.

NOTA: este editorial é publicado por CLINICS livre de restrições de copyright. Oferecemo-lo aos periódicos científicos brasileiros para reprodução integral ou parcial, Alternativamente sugerimos que apóiem esta idéia, em editoriais originais. Tais ações sinalizarão nossa vontade política de exercer o direito republicano de peticionar perante o poder público em defesa desta que é reivindicação legítima e generalizada da comunidade editorial científica brasileira. 
A tabela 1 exibe a relação desses 138 periódicos brasileiros com Cites/Document SCImago > zero, mas sem Fator de Impacto JCR-ISI. Ressalte-se que não são periódicos de impacto nitidamente mais baixo que os da coleção JCR-ISI. Os quatro primeiros apresentam impacto
$>1.00$, o que os colocaria entre os 15 melhores do Brasil. Outros dez apresentam impacto maior que 0.50 , acima da mediana dos periódicos brasileiros no JCR-ISI. Todos os 138 títulos evidentemente fariam jus à classificação 'com fator de impacto'.

Tabela 1. Cento e trinta e oito periódicos brasileiros excluídos do QUALIS com Fator de Impacto SCIMAGO (simulação 2009).

\begin{tabular}{|c|c|c|c|c|c|}
\hline Rank & Título & $\begin{array}{l}\text { cites/doc } \\
\text { scimago }\end{array}$ & Rank & Título & $\begin{array}{c}\text { cites/doc } \\
\text { scimago }\end{array}$ \\
\hline 1 & Acta Scientiarum - Agronomy & 1,19 & 48 & Revista Brasileira de Hematologia e Hemoterapia & 0,26 \\
\hline 2 & International Braz J Urol & 1,09 & 49 & Revista de Economia Politica & 0,26 \\
\hline 3 & Revista do Instituto de Medicina Tropical de Sao Paulo & 1,08 & 50 & Ceramica & 0,25 \\
\hline 4 & Brazilian Journal of Plant Physiology & 1,05 & 51 & Estudos Avancados & 0,25 \\
\hline 5 & Annual Review of Biomedical Sciences & 0,85 & 52 & Interface: Comunicação, Saude, Educação & 0,25 \\
\hline 6 & Jornal Brasileiro de Pneumologia & 0,85 & 53 & Online Brazilian Journal of Nursing & 0,24 \\
\hline 7 & Revista Brasileira de Epidemiologia & 0,73 & 54 & Boletim de Geociencias - Petrobras & 0,23 \\
\hline 8 & Brazilian Journal of Cardiovascular Surgery & 0,72 & 55 & HAHR - Hispanic American Historical Review & 0,23 \\
\hline 9 & Acta Ortopedica Brasileira & 0,71 & 56 & Opiniao Publica & 0,23 \\
\hline 10 & Brazilian Journal of Biology & 0,69 & 57 & Revista Brasileira de Ciencias Farmaceuticas & 0,23 \\
\hline 11 & Brazilian Dental Journal & 0,67 & 58 & Cadernos de Pesquisa & 0,22 \\
\hline 12 & Ciencia e Saude Coletiva & 0,58 & 59 & Anais Brasileiros de Dermatologia & 0,21 \\
\hline 13 & Materials Research & 0,55 & 60 & Journal of Public Child Welfare & 0,21 \\
\hline 14 & Brazilian Oral Research & 0,53 & 61 & Lundiana & 0,21 \\
\hline 15 & Arquivos de Gastroenterologia & 0,49 & 62 & Revista de Ciencias Farmaceuticas Basica e Aplicada & 0,21 \\
\hline 16 & Revista Brasileira de Saude Materno Infantil & 0,49 & 63 & Tropical Plant Pathology & 0,21 \\
\hline 17 & Acta Botanica Brasilica & 0,48 & 64 & Jornal Vascular Brasileiro & 0,20 \\
\hline 18 & Phyllomedusa & 0,48 & 65 & Revista Brasileira de Enfermagem & 0,20 \\
\hline 19 & Acta Scientiarum - Biological Sciences & 0,46 & 66 & Jornal Brasileiro de Patologia e Medicina Laboratorial & 0,19 \\
\hline 20 & Revista Brasileira de Medicina do Esporte & 0,46 & 67 & Historia, Ciencias, Saude - Manguinhos & 0,18 \\
\hline 21 & Revista Brasileira de Botanica & 0,45 & 68 & Revista de Psiquiatria do Rio Grande do Sul & 0,18 \\
\hline 22 & PRO-FONO: Revista de Actualização Cientifica & 0,44 & 69 & Geociencias & 0,17 \\
\hline 23 & Revista Brasileira de Engenharia Agricola e Ambiental & 0,41 & 70 & Revista Brasileira de Reumatologia & 0,17 \\
\hline 24 & Engenharia Agricola & 0,40 & 71 & Ararajuba & 0,16 \\
\hline 25 & Arquivos Brasileiros de Oftalmologia & 0,38 & 72 & Revista Brasileira de Cardiologia Invasiva & 0,16 \\
\hline 26 & Biota Neotropica & 0,38 & 73 & Revista Brasileira de Economia & 0,16 \\
\hline 27 & Radiologia Brasileira & 0,38 & 74 & Revista Brasileira de Geofisica & 0,16 \\
\hline 28 & Revista Brasileira de Otorrinolaringologia (English ed.) & 0,38 & 75 & Boletim de Ciencias Geodesicas & 0,15 \\
\hline 29 & Revista Brasileira de Sementes & 0,38 & 76 & Controle E Automação & 0,15 \\
\hline 30 & Acta Amazonica & 0,37 & 77 & Geologia USP - Serie Cientifica & 0,15 \\
\hline 31 & Ciencia Rural & 0,36 & 78 & Economia Aplicada & 0,14 \\
\hline 32 & Arquivo Brasileiro de Medicina Veterinaria e Zootecnia & 0,35 & 79 & Engenharia Sanitaria e Ambiental & 0,14 \\
\hline 33 & Pan-American Journal of Aquatic Sciences & 0,35 & 80 & IRRIGA & 0,14 \\
\hline 34 & Revista Brasileira de Ginecologia e Obstetricia & 0,35 & 81 & Psicologia e Sociedade & 0,14 \\
\hline 35 & Acta Paulista de Enfermagem & 0,34 & 82 & Revista do Colegio Brasileiro de Cirurgioes & 0,14 \\
\hline 36 & Ecletica Quimica & 0,34 & 83 & Revista Gaucha de Enfermagem / EENFUFRGS & 0,14 \\
\hline 37 & Papeis Avulsos de Zoologia & 0,33 & 84 & Educação e Pesquisa & 0,13 \\
\hline 38 & Revista Brasileira de Plantas Medicinais & 0,31 & 85 & Lua Nova - Revista de Cultura e Politica & 0,13 \\
\hline 39 & Bragantia & 0,29 & 86 & Produção & 0,13 \\
\hline 40 & Revista Arvore & 0,29 & 87 & Psicologia: Teoria e Pesquisa & 0,13 \\
\hline 41 & Revista Brasileira de Anestesiologia & 0,29 & 88 & Revista de Economia e Sociologia Rural & 0,13 \\
\hline 42 & Revista de Psiquiatria Clinica & 0,29 & 89 & Educação e Sociedade & 0,12 \\
\hline 43 & Jornal Brasileiro de Psiquiatria & 0,28 & 90 & Physis: Revista de Saude Coletiva & 0,12 \\
\hline 44 & Journal of Microwaves and Optoelectronics & 0,27 & 91 & Revista Brasileira de Ciencias Sociais & 0,12 \\
\hline 45 & Pesquisa Operacional & 0,27 & 92 & Sociologias & 0,12 \\
\hline 46 & Scientia Forestalis/Forest Sciences & 0,27 & 93 & Ambiente \& Sociedade & 0,11 \\
\hline 47 & Revista Brasileira de Ciencia Avicola & 0,26 & 94 & Brazilian Journal of Oral Sciences & 0,11 \\
\hline
\end{tabular}


Tabela 1. Continuação

\begin{tabular}{|c|c|c|c|c|c|}
\hline Rank & Título & $\begin{array}{l}\text { cites/doc } \\
\text { scimago }\end{array}$ & Rank & Título & $\begin{array}{c}\text { cites/doc } \\
\text { scimago }\end{array}$ \\
\hline 95 & Gestão e Produção & 0,11 & 117 & Revista de Sociologia e Politica & 0,06 \\
\hline 96 & Journal of Epilepsy and Clinical Neurophysiology & 0,11 & 118 & Ensaio & 0,05 \\
\hline 97 & Psicologia em Estudo & 0,11 & 119 & Estudos Ibero-Americanos & 0,05 \\
\hline 98 & Soils and Rocks & 0,11 & 120 & Novos Estudos CEBRAP & 0,05 \\
\hline 99 & Archives of Veterinary Science & 0,10 & 121 & Perspectivas em Ciencia da Informação & 0,05 \\
\hline 100 & Estudos Feministas & 0,10 & 122 & Revista Brasileira de Educação Especial & 0,05 \\
\hline 101 & Jornal Brasileiro de Reprodução Assistida & 0,10 & 123 & Revista Brasileira de Medicina & 0,05 \\
\hline 102 & Psiquiatria Biologica & 0,10 & 124 & Revista de Economia Contemporanea & 0,05 \\
\hline 103 & Anuario do Instituto de Geociencias & 0,09 & 125 & Alea & 0,04 \\
\hline 104 & Acta Scientiarum - Health Sciences & 0,08 & 126 & Horizontes Antropologicos & 0,04 \\
\hline 105 & Coluna/ Columna & 0,08 & 127 & International Journal of Atherosclerosis & 0,04 \\
\hline 106 & GED - Gastrenterologia Endoscopia Digestiva & 0,08 & 128 & Revista Brasileira de Historia & 0,04 \\
\hline 107 & Revista de Administração Publica & 0,08 & 129 & Trans/Form/Ação & 0,04 \\
\hline 108 & Revista Dental Press de Ortodontia e Ortopedia Facial & 0,08 & 130 & Cadernos Pagu & 0,03 \\
\hline 109 & Saude e Sociedade & 0,08 & 131 & Historia & 0,03 \\
\hline 110 & Summa Phytopathologica & 0,08 & 132 & Medicina & 0,03 \\
\hline 111 & Estudos de Psicologia (Campinas) & 0,07 & 133 & Psicologia Clinica & 0,03 \\
\hline 112 & Cadernos CEDES & 0,06 & 134 & Ciencia da Informação & 0,02 \\
\hline 113 & Revista Brasileira de Coloproctologia & 0,06 & 135 & Revista Latinoamericana de Psicopatologia Fundamental & 0,02 \\
\hline 114 & Revista Brasileira de Educação & 0,06 & 136 & Revista Neurociencias & 0,02 \\
\hline 115 & Revista Brasileira de Estudos de População & 0,06 & 137 & Sociedade e Estado & 0,02 \\
\hline 116 & Revista Brasileira de Oftalmologia & 0,06 & 138 & Revista Brasileira de Gestao e Desenvolvimento Regional & 0,01 \\
\hline
\end{tabular}

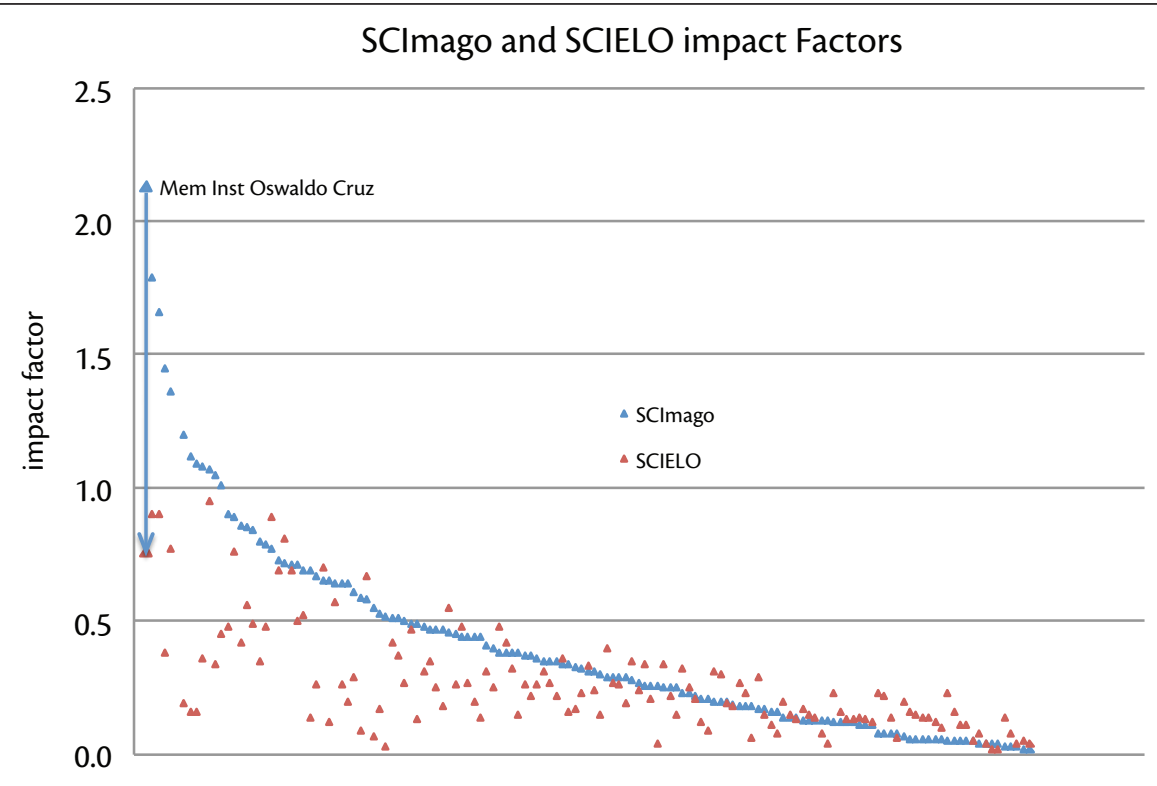

Figura 2. Fatores de Impacto SCImago e SCIELO (2009) para 142 periódicos brasileiros representados nos dois índices com valor maior que zero. Observe-se que quando SCImago > SCIELO a diferença é frequentemente grande (como indicado pela seta vertical relativa às Memórias do Instituto Oswaldo Cruz), mas invariavelmente diminuta quando SCIELO > SCImago.

Outra correlação interessante ocorre entre SCImago e SCIelo. Em primeiro lugar vale notar que existe extensa concordância: a coleção SCImago contem 235 títulos brasileiros e a coleção SCIELO contem 223. As inclusões não são 100\% concordante: a coleção SCIMAGO contem 69 periódicos ausentes da coleção SCIELO; reciprocamente a coleção SCIELO contem 56 periódicos ausentes da coleção SCImago. Por si só esta convergência revela a consistência de qualidade dos periódicos incluídos na coleção SCIELO. 
É fácil intuir que, para qualquer periódico brasileiro incluído nas duas coleções, há que se esperar que o impacto SCImago seja maior que o SCIELO, porque a coleção SCImago contem 18.732 periódicos, contra apenas 759 na coleção SCIELO. Surpreendentemente, porém, o efeito dessa enorme desproporção entre bases de dados é menor do que o esperado, como se pode ver à figura 2: dentre os 142 periódicos brasileiros presentes nas duas coleções apenas 88 (62\%) apresentam SCImago > SCIELO, enquanto 45 (32\%) apresentam SCIELO > SCImago e nove (6\%) apresentam igualdade. Esta discrepância entre o esperado e o observado merece estudo bibliométrico adicional, mas uma boa hipótese seria que artigos brasileiros citam outros artigos brasileiros com mais intensidade em virtude de um pronunciado interesse local específico. A correlação entre os impactos (Figura $3)$ é igualmente reveladora: o coeficiente angular $(0,54)$ sugere que o impacto médio SCIELO é apenas $40 \%$ menor que o impacto SCImago. Já o alto índice de correlação $\left(\mathrm{r}^{2}=0.62\right.$; $\mathrm{p}<0.01)$ demonstra que as duas métricas avaliam o mesmo parâmetro em bases de dados muito díspares.

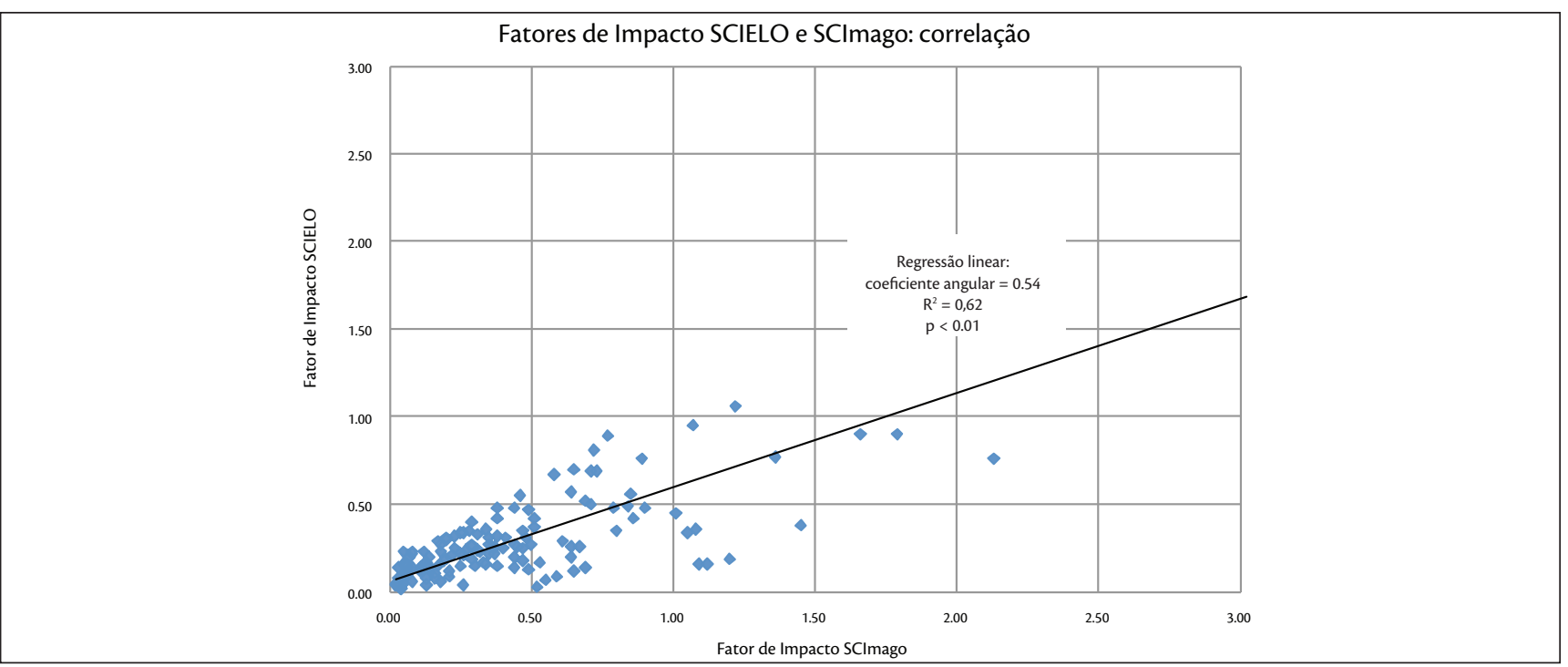

Figura 3. Correlação entre fatores do impacto SCIELO e SCImago (2009) para 142 periódicos brasileiros representados nos dois índices com valor maior que zero. O coeficiente angular indica um angulo de $28^{\circ}$ e um impacto SCIELO médio $40 \%$ menor que o SCImago. A correlação significativa indica que os dois parâmetros medem a mesma coisa sobre bases de dados díspares.

Tabela 2. Trinta e cinco periódicos brasileiros excluídos do QUALIS com Fator de Impacto SCIELO (simulação 2009).

\begin{tabular}{llc}
\hline Rank & Título & IF scielo \\
\hline 1 & Revista da Sociedade Brasileira de Fonoaudiologia & 0,81 \\
2 & Texto e Contexto Enfermagem & 0,65 \\
3 & Revista CEFAC & 0,64 \\
4 & Revista Brasileira de Educação Médica & 0,58 \\
5 & Ciência e Agrotecnologia & 0,42 \\
6 & Avaliação: Revista da Avaliação da Educação Superior & 0,28 \\
7 & Revista Paulista de Pediatria & 0,28 \\
8 & Paidéia (Ribeirão Preto) & 0,27 \\
9 & RAE Electronica & 0,21 \\
10 & Revista Brasileira de Terapia Intensiva & 0,21 \\
11 & Psicologia \& Sociedade & 0,15 \\
12 & Revista Estudos Feministas & 0,15 \\
13 & Economia e Sociedade & 0,14 \\
14 & Revista Brasileira de Meteorologia & 0,14 \\
15 & Ciência da Informação & 0,12 \\
16 & Psicologia Escolar e Educacional (Impresso) & 0,12 \\
17 & Educação em Revista & 0,11 \\
18 & Revista de Administração Contemporânea & 0,11 \\
\hline & &
\end{tabular}

\begin{tabular}{llc}
\hline Rank & Título & IF scielo \\
\hline 19 & Revista Brasileira de Ortopedia & 0,10 \\
20 & Varia Historia & 0,09 \\
21 & Matéria (Rio de Janeiro) & 0,08 \\
22 & Psicologia USP & 0,08 \\
23 & Caderno CRH & 0,07 \\
24 & DELTA Documentacao de Estudos em Linguistica Teorica & 0,07 \\
& e Aplicada & \\
25 & Ciência \& Educação (Bauru) & 0,06 \\
26 & Nova Economia & 0,06 \\
27 & Religião \& Sociedade & 0,06 \\
28 & Escola Anna Nery & 0,05 \\
29 & Estudos Economicos & 0,05 \\
30 & Revista Contabilidade \& Finanças & 0,05 \\
31 & Sba: Controle \& Automação Sociedade Brasileira de Automatica & 0,05 \\
32 & Contexto Internacional & 0,04 \\
33 & Educar em Revista & 0,04 \\
34 & Estudos de Psicologia (Natal) & 0,03 \\
35 & Interações (Campo Grande) & 0,03 \\
\hline
\end{tabular}


Caso a CAPES reconheça o Cites/Document SCImago estará resgatada a maior parte dos periódicos brasileiros com impacto $>0$. Mas a Tabela 2 mostra que, se a decisão fosse tomada neste momento restariam 35 periódicos brasileiros com impacto SCIELO maior que zero mas ausentes do JCR-ISI e do SCImago. Aqui também encontramos impactos não triviais: quatro periódicos apresentam impactos maiores que a mediana da coleção ISI. Sem esquecer a forte possibilidade de que estes 35 impactos SCIELO infra-estimem o que seriam seus impactos ISI ou SCImago.

Desta simulação, podemos concluir que teríamos 173 periódicos brasileiros com IMPACTO > ZERO tratados como "SEM FATOR DE IMPACTO” pelo QUALIS caso a avaliação fosse agora e caso os critérios 2010 fossem repetidos. Sabemos que esta "simulada exclusão" não é estática: quando a tabela Qualis "fechar” para a próxima avaliação muita coisa terá mudado: dentre elas, com certeza, as tabelas ISI-JCR, SCImago e SCIELO, bem como (esperamos!) os critérios CAPES de avaliação. Mas defendemos a tese de que reconhecer tão somente o Fator de Impacto ISI-JCR não seria lógico. Por isso entendemos que esta simulação é a base racional para um alerta, em tempo, pela adoção de novos critérios.

\section{Referências}

1. Rocha e Silva M. Qualis 2011-2013: os três erres. Clinics. 2010;65: 935-6.

2. SClmago. (2007). SJR - SCImago Journal \& Country Rank. Retrieved January 26, 2011, from http://www.scimagojr.com 